\title{
Les alternances objet $/$ oblique en français : goûter (à) un vin, dévaler (de) la colline, chercher (après) ses clés, etc. ${ }^{1}$
}

\author{
Richard Huyghe et Gilles Corminboeuf* \\ Université de Fribourg, Suisse.
}

\begin{abstract}
Résumé. L'étude porte sur le cas où deux constructions, l'une à complément d'objet, l'autre à complément oblique, sont en concurrence pour un même verbe (goûter $X /$ goûter à $X$, chercher $X /$ chercher après $X$, pousser $X /$ pousser sur $X$ ). Nous définissons dans un premier temps les conditions exactes des alternances étudiées et clarifions plusieurs points relatifs à la polysémie verbale, au statut syntaxique du SP et à l'extension d'emploi de l'alternance. Nous consacrons la seconde section du papier à l'étude des contreparties sémantiques éventuellement associées à l'existence de deux constructions distinctes. Nous établissons une tripartition des alternances, fondée sur la littérature scientifique à disposition, et nous montrons que la classification proposée ne couvre pas l'ensemble des cas répertoriés en français. Nous proposons une approche multifactorielle du phénomène, qui ménage notamment une place aux faits de variation inter-locuteurs.
\end{abstract}

\begin{abstract}
Object/oblique alternations in French. This paper deals with object/oblique alternations (OOAs) in French (e.g. goûter $X$ 'taste + NP' / goûter à $X$ 'taste + PP', chercher $X$ 'seek + NP' / chercher après $X$ 'seek + PP', pousser $X$ 'push + NP' / pousser sur $X$ 'push + PP'). We first define the exact conditions under which OOAs occur, and clarify questions about verbal polysemy, the syntactic status of the PPs involved, and selectional restrictions in alternating constructions. We then investigate the possible semantic differences triggered by OOAs, and present a ternary classification of OOAs based upon the existing literature. It appears that this very classification cannot account for all instances in French. A multifactorial approach of the phenomenon is proposed, which takes into account interspeaker variability
\end{abstract}

\section{Introduction}

La présente étude porte sur le cas où deux constructions sont en concurrence pour un même verbe :

\footnotetext{
*richard.huyghe@unifr.ch ; gilles.corminboeuf@unifr.ch
} 
(1) a. Une longue raie de cambouis, toute fraîche, barrait la face intérieure des doigts. Pourtant il n'avait pas touché à la chaîne de la bicyclette. (A. Robbe-Grillet, Frantext)

b. Le seul avantage est que si l'on touche la chaîne, pas de salissure ! (web, à propos d'un lubrifiant)

(2) L'auteur y traitait d'une question centrale (...) Le second traitait une question très proche du cours. (web, Rapport d'un jury, p. 21 et p. 42)

a. Les vagues battent contre les murs (...) (G. Flaubert, Frantext)

b. (...) la rafale bat les murs de la maison. (M. de Guérin, Frantext)

Dans (1)-(3), la construction directe du verbe alterne avec une construction oblique dans laquelle le verbe est suivi d'un SP — à conditions sémantiques et référentielles comparables.

Ce type d'alternance est étudié en détail par un certain nombre d'auteurs, en particulier en ce qui concerne l'anglais (cf. Levin 1993, Leek 1996, Broccias 2001, Adams 2001, Beavers 2006, Perek \& Lemmens 2010, Delhem 2015, inter alia). Il est également observé dans d'autres langues : Sosa Acevedo (2011) étudie le phénomène en espagnol, et Beavers (2006) mentionne des exemples en allemand, hébreu, japonais et néerlandais.

Pour ce qui est du français, la documentation du phénomène est plus sporadique. Sans prétention d'exhaustivité, on peut distinguer trois groupes de travaux. (i) Goosse-Grevisse (2016) et Blinkenberg (1960) listent une grande partie des verbes concernés. Dans une section nommée «Concurrences et substitutions » (§ 282sq), Goosse-Grevisse mentionne ainsi une centaine de verbes impliqués, tout en ménageant une place aux variations diachronique (secourir à ses vrais servants, obéir les loix), diatopique ou diastratique (regarder après 'veiller sur' est identifié comme du français populaire de Belgique), et en signalant des régularisations analogiques (pallier à sur le modèle de remédier à). Blinkenberg (1960), dans son Chapitre IX intitulé «Le spectre sémantique des groupes transitifs et la répartition des objets directs et indirects », opère également un recensement de plus de 120 verbes à alternance. L'auteur discute des cas problématiques et intègre également la dimension variationnelle, sur laquelle nous reviendrons (cf. § 2.5). Il reste que dans ces deux cas, les auteurs ne définissent pas explicitement les conditions identificatoires de l'alternance verbale observée. Les listes d'exemples établies présentent à cet égard une hétérogénéité qu'il faudrait analyser. (ii) Les contributions de Goyens (1998, 2000, 2001) portent sur le volet diachronique. Goyens montre que certains verbes qui introduisaient jadis un objet direct ont adopté une autre forme de complémentation au cours de l'histoire, et vice versa. (iii) Un troisième groupe de travaux mentionne le phénomène en passant et les auteurs proposent çà et là des hypothèses explicatives, sans accorder à ces alternances une place centrale dans l'analyse. C'est le cas par exemple de Gougenheim (1970 : 27sq), Willems (1981 : passim), Picoche (1986 : 59-61), Herslund (1997a, 1997b : 79), Riegel et al. (1994: 224-225), Bat-Zeev Shyldkrot \& Kemmer (1995: 212-213), Franckel \& Paillard (2007 : 106-107), Blanche-Benveniste (2010 : 124-125), Sablayrolles (2011 : 42), ou encore Melis (2017:29).

Il reste que les alternances du type de (1)-(3) demeurent relativement méconnues. D’une part, il n'existe pas en français de liste exhaustive des verbes qui autorisent l'alternance, ni a fortiori d'analyse sémantique et de classification de ces verbes. D'autre part, il semblerait que certaines alternances, contrairement à d'autres, présentent une contrepartie sémantique. Par exemple, pallier une défaillance et pallier à une défaillance ne comportent pas de différence de sens apparente, tandis que grimper la falaise et grimper sur la falaise se distinguent par l'expression du chemin parcouru ou de la destination. Ces différences de corrélation sémantique demandent à être expliquées.

Nous présentons dans ce travail une première analyse de l'alternance entre constructions verbales à complément objet ou oblique. Notre objectif est de déterminer les conditions 
exactes de cette alternance et d'effectuer une première série d'observations sur les variations de sens et d'usage qu'elle occasionne. Ce travail est envisagé comme préparatoire au recensement des verbes qui permettent l'alternance en français et à l'analyse sémantique de chaque cas d'alternance recensé.

L'article comporte deux sections. La première s'attache à définir précisément les cas d'alternance étudiés, et clarifie certains points relatifs à la polysémie des verbes concernés, au statut syntactico-sémantique des constituants qui alternent, et à l'extension d'emploi de l'alternance pour un verbe donné. La seconde section est consacrée à l'étude des différences de sens éventuellement associées à l'existence de deux constructions distinctes. Nous proposons in fine une approche multifactorielle du phénomène, qui ménage une place aux faits de variation inter-locuteurs.

\section{Conditions d'alternance}

Nous étudions ici les alternances de construction entre objets directs et obliques, pour un verbe et un contenu lexical donnés. Par «obliques», nous désignons les arguments syntaxiques ou sémantiques du verbe qui ne sont ni sujets ni cliticisables par le / la / les. Les alternances de ce type observées en français interviennent généralement entre une construction directe et une construction prépositionnelle. Nous appelons ainsi « alternance objet / oblique » (AOO) la relation entre deux constructions A et B qui satisfont l'ensemble des conditions suivantes :

- A est de la forme $\mathrm{SN}_{1} \mathrm{~V}_{1} \mathrm{SN}_{2}$,

- B est de la forme $\mathrm{SN}_{3} \mathrm{~V}_{2}$ Prép $\mathrm{SN}_{4}$,

-il existe au moins une occurrence de $\mathrm{A}$ et une occurrence de $\mathrm{B}$ dans lesquelles à la fois $\mathrm{V}_{1}$ et $\mathrm{V}_{2}, \mathrm{SN}_{1}$ et $\mathrm{SN}_{3}, \mathrm{SN}_{2}$ et $\mathrm{SN}_{4}$ sont identiques,

- $\mathrm{SN}_{2}$ est l'objet direct de $\mathrm{V}_{1}$,

- Prép $\mathrm{SN}_{4}$ est un argument syntaxique ou sémantique de $\mathrm{V}_{2}$,

$-V_{1}$ et $V_{2}$ sont employés sans variation de voix ${ }^{2}$.

On peut ajouter qu'en cas d'alternance, $\mathrm{A}$ et $\mathrm{B}$ renvoient généralement à deux situations du monde proches ou équivalentes.

Nous nous limitons dans ce travail aux cas d'alternance entre constituants nominaux. On peut noter qu'une grande partie des alternances de surface entre arguments propositionnels directs et indirects ne constituent pas des cas d'AOO, puisque la cliticisation est la même dans les deux variantes :

(4) a. Je me réjouis (de ce) que vous soyez venus.

b. Je $\{$ m'en $/ *$ me le $\}$ réjouis.

Par ailleurs, si l'alternance peut se manifester sous forme pronominale :

(5) a. sans bidouiller le vin on va pouvoir lui aider à évoluer (oral radio)

b. À chaque « régime » semble correspondre par ailleurs une manière spécifique, pour une société, d'envisager son passé, d'en traiter et de le traiter. (web)

il convient de préciser que cette double construction ne s'observe pas pour les mêmes verbes selon que les constituants qui commutent sont des SN / SP ou des clitiques :

(6) a. Ça $\{$ le / lui $\}$ regarde.

b. Ça regarde Pierre.

c. ??Ça regarde à Pierre.

(7) a. Une fureur le prend. (G. Flaubert, Frantext)

b. Parfois une rancœur lui prend. (J. Vallès, Frantext)

c. Une \{fureur / rancœur\} prend (??à) ce personnage. 
Les clitiques le et lui de (6)-(7) ne semblent pas avoir de correspondants lexicaux à droite $\mathrm{du}$ verbe. La proximité phonologique des clitiques de troisième personne (le et lui, les et leur), combinée à l'indistinction des formes datives et accusatives aux première et deuxième personnes, semble faciliter l'alternance pronominale. Le phénomène diffère en partie de celui observé avec les compléments lexicaux. Pour la même raison, nous ne traiterons pas ici des cas d'alternance entre pronoms dans la construction causative en faire (les / leur faire broyer du noir, cf. Lamiroy \& Charolles 2011).

Nous écartons également de notre étude les cas où l'alternance entre SN et SP s'effectue en relation avec un autre argument verbal, comme dans la double construction des $\mathrm{V}$ de 'chargement' (8a) (cf. Fillmore 1968, Danlos 1986, Guillet \& Leclère 1992, Beavers 2006, inter alia), les configurations à attribut de l'objet (8b) ou les structures de possession inaliénable (8c) - l'alternance n'étant ici possible qu'en cas d'ellipse de l'objet (Il embrasse Marie \{dans / *ø\} le cou).

(8) a. Pierre a chargé \{des bûches sur le camion / le camion de bûches\}.

b. Ils ont nommé une femme (comme) chef du gouvernement.

c. Il embrasse (dans) le cou.

Dans ce premier travail, nous nous concentrons donc sur la relation qui existe entre le $\mathrm{V}$ sélectionné et la forme prépositionnelle ou non de son complément. De ce point de vue, la définition de l'AOO, telle qu'elle est énoncée ci-dessus, nécessite plusieurs clarifications.

\subsection{Identité verbale}

L'identité de $V_{1}$ et de $V_{2}$ en cas d'alternance repose sur deux conditions. La première est que $\mathrm{V}_{1}$ et $\mathrm{V}_{2}$ aient le même paradigme flexionnel. Des verbes comme descendre et monter n'alternent pas aux temps composés s'ils s'emploient dans A et B avec des auxiliaires différents :

(9) a. Marion a descendu l'escalier.

b. Marion est descendue de l'escalier. ${ }^{3}$

La seconde condition est sémantique. Elle stipule que $V_{1}$ et $V_{2}$ correspondent à une même acception en cas de polysémie ou d'homonymie verbale. Sont ainsi exclues les alternances formelles dénuées de proximité sémantique :

(10) a. Sophie aspire le bon air de la montagne.

b. Sophie aspire au bon air de la montagne.

Qu'aspirer 'inhaler' et aspirer 'désirer' puissent accidentellement sélectionner un même objet n'en fait pas un $\mathrm{V}$ unique présentant l'alternance objet / oblique. De même, ne ressortissent pas à l'AOO les doubles constructions telles que déboucher (sur) une canalisation, tenir (à) ses promesses, tomber (sur) des filles.

La condition d'équivalence sémantique entre $V_{1}$ et $V_{2}$ implique notamment que le rôle sémantique du sujet doit être maintenu en cas d'alternance. Le changement de rôle du sujet dans (11) — agent dans (11a), patient dans (11b) — suffit à exclure guérir (de) de l'AOO :

(11) a. Pierre a guéri une maladie.

b. Pierre a guéri d'une maladie.

On peut noter que certains verbes polysémiques n'alternent que dans une de leurs acceptions. Tel est le cas d'accoucher qui n'admet la double construction que dans son emploi non factitif (13)-(14) :

(12) a. La sage-femme a accouché la parturiente.

b. \#La sage-femme a accouché de la parturiente. 
(13) a. Elle a accouché une petite fille, et elle va l'appeler Mei. (web)

b. Elle a accouché d'une petite fille, et elle va l'appeler Mei.

(14) a. L'idéal hygiéniste accouchait l'utopie inquiétante d'un monde purgé des souillures (...) (A.-M. Garat, Frantext)

b. À la veille d'Internet, des enfants de Gutenberg voulaient accoucher d'un monde de cosmonautes (...) (R. Debray, Frantext)

De même, contrairement à goûter ${ }_{1}$ 'expérimenter', goûter ${ }_{2}$ 'apprécier' ne présente pas d'alternance objet / oblique, - et ce quelle que soit sa communauté sélectionnelle avec goûter $_{1}$ :

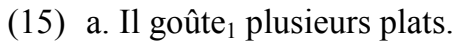

b. Il goûte 1 à plusieurs plats.

(16) a. Il goûte ${ }_{2}$ les joies de la glisse.

b. *Il goûte ${ }_{2}$ aux joies de la glisse.

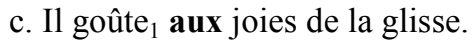

Dans certains cas, il peut être difficile de dire si la condition d'équivalence sémantique entre $V_{1}$ et $V_{2}$ est satisfaite. Comme nous le verrons plus loin ( $\S 2$.), l'alternance entre les constructions directe et oblique est parfois corrélée à une différence de sens. Aussi convient-il de s'interroger sur l'origine de la variation sémantique observée entre deux énoncés incluant un même argument précédé ou non d'une préposition : cette variation estelle le fait de l'ensemble de la construction (avec / sans préposition) ou d'un changement de sens du V (auquel cas la condition d'identité entre $\mathrm{V}_{1}$ et $\mathrm{V}_{2}$ ne serait pas respectée) ? La

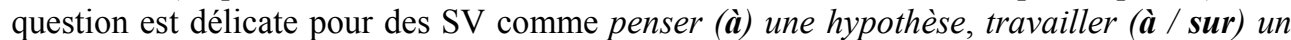
article, tirer (sur) un renard. Elle ne l'est toutefois qu'en cas de grande proximité sémantique voire de quasi-synonymie entre $V_{1}$ et $V_{2}$, par opposition aux faits de polysémie ou d'homonymie manifestes. Par défaut, nous considérerons comme AOO les occurrences de double construction dans lesquelles on peut penser que la différence de sens est générée par l'ensemble de la construction $V+S N / S P$, et non par le V lui-même.

\subsection{Statut du SP}

Les alternances étudiées mettent en jeu deux réalisations différentes d'un même argument verbal, que celui-ci soit syntaxique ou sémantique. Il est connu que le statut syntaxique des compléments prépositionnels peut être difficile à déterminer, eu égard à la distinction entre arguments et ajouts. On peut de ce point de vue distinguer deux pôles parmi les cas considérés comme AOO.

Le premier pôle est constitué des AOO dans lesquelles le SP (i.e. le groupe Prép $\mathrm{SN}_{4}$ ) est un argument syntaxique du verbe. Cette situation s'observe pour des verbes tels que :

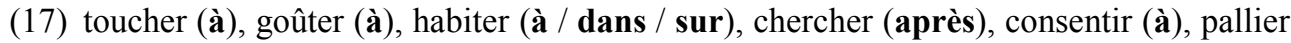

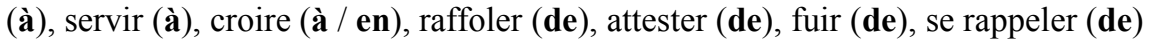

Certains des tests habituellement mobilisés pour mettre en évidence les arguments syntaxiques sont vérifiés par les SP correspondants. Par exemple, les SP argumentaux qui se construisent avec les V d'action ne peuvent pas l'être avec le faire en reprise verbale (cf. Prandi 1987, Bonami 1999) :

(18) a. *Pierre a touché, et il l'a fait à la statuette.

b. *Ceux qui ont cherché l'ont fait après leurs clés.

c. *Certains ont goûté au vin, et d'autres l'ont fait à la bière. 
De même, des V comme consentir, pallier, raffoler introduisent des SP par l'intermédiaire de prépositions qu'ils sélectionnent en les spécifiant, ce qui est un autre indice du statut argumental des SP en question ${ }^{4}$ :

(19) a. L'acteur a consenti à une baisse de son cachet.

b. *L'acteur a consenti \{de / en / sur / après / etc. $\}$ une baisse de son cachet.

Le second pôle rassemble les AOO dans lesquelles le SP paraît avoir les caractéristiques d'un ajout, comme dans les alternances suivantes :

(20) perquisitionner (à / dans), charger (sur / contre $)^{5}$, gagner (sur / contre), postuler (à / pour / sur), plaider (pour / en faveur de)

Il est possible en l'occurrence d'employer les SP avec le faire en reprise verbale, ce qui est considéré comme une condition suffisante d'identification des ajouts :

(21) a. Les forces de l'ordre ont chargé, et elles l'ont fait contre des manifestants qui voulaient voter.

b. Ceux qui ont postulé l'ont fait sur différents postes.

c. Certains enquêteurs ont perquisitionné dans la chambre, d'autres l'ont fait dans le grenier.

On peut néanmoins convenir que les V sous (21) présentent une alternance argumentale, si l'on considère qu'ici le SP renvoie à un argument sémantique du V. Par argument sémantique, nous désignons tout constituant qui dénote un participant distinctif de la situation minimale décrite par le $\mathrm{V}$, i.e. un participant dont le rôle est spécifiquement impliqué par le type de procès décrit. Ainsi perquisitionner implique-t-il sémantiquement un lieu à parcourir, charger (dans le sens concerné) une cible visée, plaider une cause à défendre, etc. Par ailleurs, la possibilité d'employer les deux constructions, directe ou prépositionnelle, en référence à une même situation étaye leur rapprochement :

(22) parce qu'y a deux types de reines hein si on veut + de gagnantes + euh y a celle de l'alpage celle qui gagne euh: toutes les autres sur les cent jours et y a la reine justement des matchs de reines + qui gagne toutes les autres mais sur un jour (oral, Ofrom ; à propos de combats de vaches, les 'reines')

Dans (22), l'insertion de la préposition contre après chaque occurrence de gagne ne produirait pas de nuance sémantique.

En revanche, ne relèvent pas de l'alternance argumentale les appariements de surface dans lesquels les SP ne dénotent pas des participants fondamentaux des procès décrits, mais peuvent avoir le même contenu lexical que les SN objets dans l'emploi direct. Les constructions avec des SP très périphériques, tels que les cadratifs spatio-temporels, ne participent généralement pas de l'AOO :

(23) « Ben Laden est déterminé à frapper aux Etats-Unis» (et non « les Etats-Unis »), comme l'a dit le porte-parole, le 17 mai. (Le Canard Enchaîné, 22.05.2002)

L'alternance des séquences frapper aux Etats-Unis et frapper les Etats-Unis est accidentelle. Elle tient au fait (i) que le V peut être employé intransitivement, i.e. sans expression de son argument interne, (ii) que ce $\mathrm{V}$ peut sélectionner comme objet une expression dénotant un territoire, encline par là même à figurer en position de cadratif locatif. Dans l'emploi prépositionnel, ce complément de lieu ne constitue pas selon notre définition un argument sémantique du V - frapper impliquant spécifiquement un agent et un patient, mais pas un localisateur. La possibilité d'insérer dans la construction prépositionnelle, à interprétation constante, un SN argumental doté du même rôle sémantique que l'objet dans la construction directe (ex. frapper une cible aux Etats-Unis)

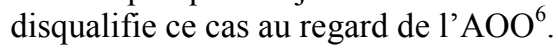


La condition de l'argument sémantique permet de la même manière d'exclure les cas d'apparentes alternances à valeurs contradictoires, comme dans réclamer (contre) une mesure. Le V réclamer implique sémantiquement un objet requis, mais pas l'opposition à une entité tierce. Le SP introduit par contre n'est donc pas un argument sémantique du V, et ne participe pas d'une $\mathrm{AOO}$ (contrairement à réclamer après $X$, où le SP est un argument sémantique).

Dans certains cas, le statut syntaxique du SP qui alterne formellement avec un SN paraît difficile à déterminer. Entre les deux pôles syntaxiques distingués ci-dessus se rencontrent des cas incertains, selon que les tests suggérés ne s'appliquent pas aisément (ex. le SP en sur compatible avec impacter peut-il ou non se construire avec le faire en reprise du V ?) ou qu'ils conduisent à des diagnostics contradictoires (ex. le SP complément de combattre paraît extractible dans une reprise en le faire, mais la préposition contre semble sélectionnée par le $\mathrm{V}$, ce qui est une propriété exclusive des arguments syntaxiques). Nous considérerons que ces cas relèvent de l'AOO dès lors que les SP en jeu constituent des arguments sémantiques du $\mathrm{V}$, i.e. dès lors qu'ils dénotent des participants élémentaires requis dans le type de procès décrit par le verbe.

\subsection{Extension de l'alternance}

Telle qu'elle est définie ci-dessus, l'AOO met en relation deux constructions. La possibilité d'alternance dépend en premier lieu du V employé, mais celui-ci n'en détermine pas seul les conditions: l'alternance s'établit en contexte, en fonction du contenu lexical des arguments présents. Comme le note Broccias (2001) au sujet des alternances de construction verbale avec ou sans at en anglais, les possibilités d'alternance pour un $\mathrm{V}$ donné peuvent dépendre des arguments exprimés.

En français, l'extension de l'alternance varie selon que les V l'autorisent pour l'ensemble de leur spectre sélectionnel, ou pour une partie seulement. L'extension est maximale pour des $\mathrm{V}$ tels que :

(24) chercher (après), pallier (à), se rappeler (de), combattre (contre), dévaler (de), méditer (sur), anticiper (sur)

Dans l'acception concernée par l'alternance, ces verbes se prêtent à l'AOO quel que soit le contexte. Par exemple, chercher commute avec chercher après, que l'argument direct ou oblique dénote un artefact, un lieu, un animé, un objet informationnel, un état, etc. La préposition après est omissible dans chacun des exemples suivants :

(25) a. Une heure passe, le cours s'achève et le voilà qui cherche après ses clés. (web)

b. Arrivés à Baktapur, nous avons cherché après l'entrée du Durbar square. (web)

c. Ce malheureux cherche après sa soeur. (web)

d. J'ai cherché après une solution mais je n'ai rien trouvé de concluant. (web)

e. On cherche après l'amour et on est prêt à tout! (E. Courtois, paroles de chanson, web)

Lorsqu'elle s'applique à l'ensemble des emplois d'un lexème verbal, l'AOO reste dépendante des capacités sélectionnelles de ce lexème. Certains V permettent une extension maximale de l'alternance, mais pour un spectre d'emploi a priori très restreint. Par exemple, le V transitif cligner s'emploie quasiment exclusivement avec l'objet les yeux, qui alterne toujours avec le SP des yeux. De même, le V hocher sélectionne comme objet des N de parties du corps (la tête, mais aussi le menton, les épaules, la queue, etc.) :

(26) a. Décontenancé, Alexandre hoche les épaules et s'installe au volant. (web)

b. Quand nous avons fait la remarque au serveur, il a juste hoché des épaules. (web) 
Dans d'autres cas de figure, l'alternance est cantonnée à une partie seulement des types sémantiques argumentaux admis dans les deux constructions du verbe. Seules certaines classes sémantiques d'arguments sont communes aux deux emplois du verbe. L'alternance pour un $\mathrm{V}$ comme perquisitionner est partielle, en cela qu'elle ne se réalise naturellement que lorsque l'objet dénote un lieu :

(27) a. La police perquisitionne le domicile d'un député vaudois. (Tribune de Genève, 14.02.2017)

b. La police perquisitionne au domicile du pilote du Boeing 777. (Le Temps, 15.03.2014)

Sans être impossible, l'alternance se révèle nettement moins bien attestée lorsque l'objet dénote un humain :

(28) a. La police a perquisitionné chez le criminologue Alain Bauer. (Le Monde, 16.01.2017)

b. ?La police a perquisitionné le criminologue Alain Bauer.

Par ailleurs, certaines contraintes non lexicales, tenant par exemple au déterminant sélectionné et à son interprétation, peuvent intervenir dans la possibilité d'alterner les constructions en contexte. Ces contraintes s'ajoutent aux restrictions sélectionnelles et peuvent se manifester pour un type lexical donné. Ainsi espérer en n'alterne avec espérer, pour un même type d'objet, que lorsque le syntagme argumental est indéfini et spécifique (30), et non défini et générique (29) :

(29) a. Ils espèrent en \{la vie / l'avenir\}.

b. ??Ils espèrent \{la vie / l'avenir\}.

(30) a. Ils espèrent en \{une vie meilleure / un avenir radieux\}.

b. Ils espèrent \{une vie meilleure / un avenir radieux\}.

Il apparaît ici que l'AOO dépend de conditions de forme et de contenu pesant sur les arguments verbaux, qui déterminent les capacités d'alternance des $\mathrm{V}$ en contexte.

\subsection{Exemples d'alternances}

Les conditions d'alternance précisées ci-dessus nous permettent d'identifier une série de verbes qui, dans certains de leurs emplois au moins, se prêtent à l'AOO. Nous n'avons pas à ce stade établi la liste complète des verbes qui autorisent l'AOO en français. Nous nous en tenons ici à la présentation d'un échantillon de verbes à alternance :

(31) aborder (à), appuyer (sur), applaudir (à), approcher (de), attester (de), cligner (de),

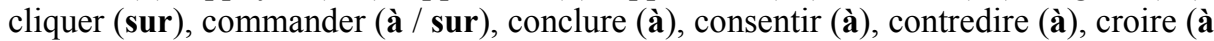
/ en / dans), déborder (de / sur), dévaler (de), discuter (de / sur), économiser (sur), empiéter (sur), encourager (à), fouiller (dans), frapper (à / contre / dans / sur), fuir (de / devant), gagner (contre), goûter (à / de), grimper (sur), habiter (à / dans / sur), hériter (de), heurter (à / contre), hocher (de), influer (sur), juger (de), lorgner (sur / vers), méditer (sur), obéir (à), parer (à), pénétrer (dans), percuter (contre), postuler (à / pour / sur), pousser (à / contre / sur), présider (à), primer (sur), raffiner (sur), réclamer (après), regarder (à), se remémorer (de), ressembler (à), shooter (dans), souscrire (à), sprayer (sur), suppléer (à), témoigner (de), tirer (sur), toquer (à / sur / contre), toucher (à / de), traiter (de) travailler (à / sur), viser (à / dans / sur), voisiner (avec), zoomer (sur), etc.

La majorité de ces verbes sont mentionnés dans la littérature (Blinkenberg 1960, GoosseGrevisse 2016); des repérages dans des corpus 'tout-venant' (presse, littérature, web, 
français parlé), de même qu'un recensement automatique dans la ressource Dicovalence (Eynde \& Mertens 2003), nous ont permis de compléter les listes existantes.

Nous avons bien sûr listé les verbes concernés sans parti pris normatif, que les alternances qu'ils présentent soient communes (goûter le vin / goûter au vin) ou éventuellement marquées (demander après quelqu'un, plier sur les genoux, surfer $\boldsymbol{\text { o }}$ une vague, obéir $\boldsymbol{\sigma}$ ses parents; $\mathrm{cf}$. 2.5 . pour un aperçu de cette dimension variationnelle).

L'examen des cas d'alternance appelle deux commentaires. D'une part, les constructions diffèrent selon que l'AOO, pour un V donné, met en jeu une ou plusieurs prépositions. Dans le second cas, la variation prépositionnelle peut dépendre du type ou du rôle sémantique de l'argument. Tandis qu'un V comme postuler semble alterner indifféremment avec les prépositions à, sur et pour, le $\mathrm{V}$ fuir commute avec fuir de ou fuir devant lorsque l'argument dénote, respectivement, un lieu d'origine ou une entité à laquelle on souhaite se dérober - en conséquence de quoi, l'alternance avec un argument dénotant un animé n'est possible qu'avec devant.

D'autre part, on peut se demander s'il existe une construction dominante dans l'alternance, relativement à la présence ou à l'absence de la préposition. Il est parfois possible d'identifier, parmi les deux expressions qui alternent, une construction standard, majoritaire dans l'usage, et généralement dotée d'un spectre de sélection plus large. Cette tournure privilégiée est le plus souvent la construction directe (ex. réclamer vs réclamer après, consulter vs consulter avec, contester vs contester contre), mais il arrive aussi qu'il s'agisse de la construction prépositionnelle, comme dans cliquer (sur), déblatérer (contre), empiéter (sur), gagner (contre), zoomer (sur) :

(32) a. Cliquez le menu Démarrer et vérifiez que les outils d'administration sont bien présents. (web)

b. Le PS a systématiquement déblatéré la droite, quand cette dernière était au pouvoir (web)

c. Nous avons zoomé le carnet du lieutenant Beauregard. (G. Raynaud, Google Livres)

La conversion ici d'un oblique en argument prototypique (si l'on considère comme tels les objets directs) est remarquable. Une étude spécifique pourrait être consacrée aux raisons profondes qui conduisent en l'occurrence à l'abandon de la préposition — et qui ont sans doute à voir avec la saillance sémantique de l'argument dans le procès décrit.?

\section{Corrélations sémantiques}

On peut s'interroger sur les motifs d'existence de l'AOO en français, et sur une éventuelle contrepartie sémantique qui justifierait l'existence de deux constructions distinctes. Les travaux existants sur d'autres langues que le français, en particulier sur l'anglais, identifient trois types d'AOO que l'on pourrait retrouver en français. Il s'agit des cas que Levin (1993) nomme «conative alternation", «locative preposition drop alternation" et "search alternation", et que nous appellerons ici respectivement alternances "conative», « locative » et « explorative ». Nous étudions ces trois cas dans cette section, de même que le schéma d'explication unitaire que propose Beavers (2006) pour en rendre compte. Il sera établi d'une part que les alternances observées en français ne se réduisent pas aux types conatif, locatif et exploratif, et d'autre part que l'hypothèse de Beavers, bien que souvent vérifiée, n'explique pas l'ensemble des variations sémantiques corrélées à l'AOO. Nous esquisserons un traitement multifactoriel de l'AOO, qui prend en considération le phénomène dans ses diverses dimensions variationnelles. 


\subsection{Alternance conative}

L'alternance conative (lat. cōnor, cōnārī 'essayer') est décrite pour certaines constructions en $a t$ et on de l'anglais (Levin 1993, Leek 1996, Adams 2001, Beavers 2006, Delhem 2015) et certaines constructions en $a$ de l'espagnol (Sosa Acevedo 2011). Cette alternance est définie par le fait que l'emploi prépositionnel, contrairement à l'emploi direct, indique simplement l'intention d'accomplir une action donnée, sans spécifier si le but visé a été atteint :

(33) a. Paula hit the fence.

'Paula a frappé la barrière'

b. Paula hit at the fence.

'Paula a donné un coup en direction de la barrière'

Le groupe V + SP dans (33b) exprime une action entreprise, sans préciser si celle-ci a ou non abouti ${ }^{8}$ - par implicature, la phrase s'emploie plutôt, de fait, pour indiquer que l'action n'a pas atteint son but. Ce type d'alternance s'observe en anglais et en espagnol pour certains V de contact par impact (hit 'frapper', golpear 'frapper', tocar 'toucher'), de dégradation matérielle (cut 'couper', slash 'lacérer'), de pression / traction (push 'pousser', pull 'tirer'), d'ingestion (eat 'manger', chew 'mâcher') ou de perception (mirar 'regarder') ${ }^{9}$.

L'alternance conative se rencontre en français pour les V pousser et tirer qui, dans leur emploi avec ou sans préposition, connaissent la même variation d'interprétation que les $\mathrm{V}$ équivalents en anglais (dans l'emploi avec on tout au moins) :

(34) a. Pierre a poussé (sur) la table.

b. Pierre a tiré (sur) le casier.

Ici, le déplacement des objets manipulés est impliqué dans la construction directe, mais pas dans la construction prépositionnelle, ce qui explique la différence de compatibilité avec un complément de but constatée dans (35) :

(35) a. Pierre a poussé la table contre le mur.

b. \#Pierre a poussé sur la table contre le mur.

Le V tirer dans le sens de 'actionner une arme à feu', selon qu'il s'emploie avec ou sans sur, permet également l'alternance conative :

(36) Le chasseur a tiré (sur) un lapin.

Comme le signalent Franckel et Paillard (2007: 106-107), tirer un lapin indique que le lapin est abattu, ou tout au moins touché par le projectile ${ }^{10}$, tandis que tirer sur un lapin peut exprimer une action non aboutie, au sens où le tir peut avoir manqué sa cible.

Il reste que l'alternance conative est moins répandue en français qu'en anglais. On peut noter que certains équivalents en français des $\mathrm{V}$ anglais et espagnols qui admettent l'alternance conative présentent eux-mêmes une AOO, mais sans l'effet de décalage sémantique caractéristique des alternances conatives. Ainsi frapper peut-il alterner avec frapper dans, sans que l'emploi prépositionnel indique que l'objet visé n'est pas atteint par le coup :

(37) a. Le physicien peut expliquer ce qu'il se passe dans un muscle lorsqu'on frappe un ballon. (Le Temps, 13.10.2017)

b. N'oublions pas que lorsqu'on frappe dans un ballon, il y a un simple déplacement d'air. (web)

De même, l'alternance conative relevée par Sosa Acevedo (2011) pour l'espagnol mirar (a) ('regarder (en direction de)') s'observe difficilement en français. L'emploi du verbe lorgner 
avec la préposition sur ((38b) vs (38a)) ne se distingue pas par la description d'une situation dans laquelle le contact visuel n'est pas établi :

(38) a. Elle repousse la soucoupe et de nouveau lorgne sa montre. (M. Debriffe, Google Livres)

b. Arrivé sur la pointe des pieds, le président Challandes lorgne sur sa montre et repart enfiler son bleu de travail au château. (Le Temps, 26.06.2016)

S'il existe une nuance sémantique ou une différence d'interprétation entre les constructions frapper et frapper dans, lorgner et lorgner sur, celle-ci ne tient pas au décalage entre l'intentionnalité et le résultat produit par l'action. Elle n'est donc pas de type conatif.

\subsection{Alternance locative}

L'alternance locative (« locative preposition drop alternation » chez Levin) concerne les V de déplacement qui peuvent s'employer directement ou indirectement avec un complément exprimant le but, l'origine ou le chemin. Ce type d'alternance est illustré en anglais par des exemples comme (39)-(41) (cf. Levin 1993 : 43-44) :

(39) a. Martha climbed the mountain.

'Martha a gravi la montagne'

b. Martha climbed up the mountain.

'Martha a grimpé dans la montagne'

(40) a. Martha slowly descended the stairs.

'Martha a lentement descendu les escaliers'

b. Martha slowly descended down the stairs.

'Martha est lentement descendue dans les escaliers'

(41) a. They skated the canals.

'Ils ont parcouru les canaux en skate'

b. They skated along the canals.

'Ils ont fait du skate le long des canaux'

Il est généralement admis que ce type d'alternance est lié à une interprétation holistique ou non holistique de l'élément locatif : contrairement à la construction directe, l'emploi avec up, down et along ne spécifie ni que le chemin est parcouru dans son intégralité, ni que le but est nécessairement atteint.

En français, l'AOO locative s'observe pour certains verbes de déplacement employés avec des compléments de localisation qui, lorsqu'ils sont introduits par une préposition, indiquent le but ou l'origine :

(42) a. Chloé a grimpé (sur) la colline.

b. Chloé a dévalé (de) les escaliers.

Il existe ici une différence importante avec les alternances de l'anglais, qui apparaît notamment dans (42a) vs (39). Si la construction directe dans (42a) présente bien le repère de localisation comme un chemin parcouru par le sujet, la construction prépositionnelle, contrairement à ce qu'on observe dans (39b), indique que le but est nécessairement atteint. Cette différence d'interprétation est liée au statut grammatical différent de $u p$ et de sur, et aux différences profondes qui existent entre le français et l'anglais dans l'expression de la trajectoire (cf. Talmy 2000). En français, l'alternance de construction repose essentiellement sur une différence de profilage de l'élément locatif - comme chemin dans la construction directe et comme point d'arrivée avec $s u{ }^{11}$. La même différence de profilage s'observe dans (42b) : le localisateur est envisagé comme un site parcouru dans la construction directe et comme un point d'origine dans l'emploi prépositionnel. 
Ajoutons que l'existence d'AOO locatives qui, dans la construction oblique, indiquent un chemin est moins manifeste en français qu'en anglais. Les exemples (43)-(44) pourraient relever de ce type :

(43) a. Julien a sauté la barrière.

b. Julien a sauté \{au-dessus de / par-dessus\} la barrière.

(44) a. Quand on surfe une vague de 30 mètres, cela se joue entre la peur et l'envie. (web)

b. Un Français surfe sur une vague de plus de 30 mètres de haut au Portugal. (web)

Le statut d'argument (syntaxique ou sémantique) des constituants prépositionnels est plus discutable dans ces exemples que dans les cas précédents. On peut noter que la construction dominante avec le V surfer est prépositionnelle, l'emploi transitif (44a) paraissant plus rare (et peut-être promu par les spécialistes et adeptes du sport concerné). Il n'apparaît pas, dans les deux paires d'exemples ci-dessus, de différence claire en termes d'extension du parcours décrit. En particulier, la construction prépositionnelle ne semble pas moins holistique dans son interprétation que la construction directe. Quelle que soit la tournure sélectionnée dans (43), le franchissement décrit est intégral, et les exemples (44) sont sousdéterminés quant à l'extension du chemin parcouru, sans qu'une variante paraisse à cet égard plus spécifiée que l'autre.

\subsection{Alternance explorative}

L'alternance explorative concerne en anglais des V comme search (Levin 1993: 70), qui expriment une action d'investigation et présentent la double construction $\mathrm{SN}_{1} \mathrm{~V} \mathrm{SN}_{2}$ for $\mathrm{SN}_{3}$ et $\mathrm{SN}_{1} \mathrm{~V}$ for $\mathrm{SN}_{3}$ in $\mathrm{SN}_{2}$ (ou parfois $\mathrm{SN}_{1} \mathrm{~V} \mathrm{SN}_{3}$ in $\mathrm{SN}_{2}$ ) :

(45) a. Melissa searched the papers for a clue.

'Melissa a parcouru les documents à la recherche d'un indice'

b. Melissa searched for a clue in the papers.

'Melissa a cherché un indice dans les documents'

Selon Beavers (2006 : 74), l'emploi direct du $\mathrm{SN}_{2}$ se distingue de l'emploi oblique par la description d'une situation où le référent est exploré dans sa totalité. L'effet observé est comparable à celui de l'alternance locative de l'anglais, en cela que seule la construction directe indique un parcours exhaustif de l'entité dénotée par le constituant en alternance.

En français, des V comme fouiller, inspecter, perquisitionner, fourrager présentent une capacité d'alternance proche de celle relevée pour les $\mathrm{V}$ comme search :

(46) a. J'ai fouillé l'armoire à pharmacie, mais il n'y avait rien. (web)

b. Isidore fonce dans la salle de bains, fouille dans l'armoire à pharmacie, trouve le tube blanc et en tire un cachet qu'il apporte avec un verre d'eau. (B. Werber, Google Livres)

L'alternance explorative en français ne met en jeu que deux arguments, et non trois comme en anglais. On retrouve néanmoins la même variation d'interprétation entre les deux tournures : la construction directe (46a) se distingue de la construction prépositionnelle (46b) en ce qu'elle décrit une exploration complète du site investigué. Cette différence d'interprétation explique que la lecture télique soit favorisée dans l'emploi transitif (Herslund 1997a : 61) :

(47) a. La police a fouillé l'appartement en vingt-cinq minutes.

b. ?La police a fouillé dans l'appartement en vingt-cinq minutes.

On peut remarquer que les verbes d'exploration dont l'objet direct ne peut pas exprimer un lieu de recherche se comportent différemment des V comme search et fouiller. Tel est le 
cas du V chercher, qui se prête à l'AOO, mais dont l'argument direct ne peut dénoter qu'un objet de recherche :

(48) a. Pierre cherche (après) ses clés (dans l'appartement).

b. *Pierre cherche l'appartement après ses clés.

La construction $\mathrm{SN}_{1} \mathrm{~V} \mathrm{SN} \mathrm{SN}_{2}$ Prép $\mathrm{SN}_{3}\left(\mathrm{SN}_{2}\right.$ et $\mathrm{SN}_{3}$ dénotant respectivement le lieu exploré et l'entité recherchée) est exclue ici. Il n'y a pas d'équivalence entre les propriétés de construction de chercher et celles de search et fouiller. Il s'ensuit que l'alternance pour chercher n'est pas corrélée à une différence d'information quant au parcours effectué. En outre, dans (48a) aucune différence d'interprétation holistique selon la version employée n'est observable. Il n'y a pas, par exemple, de différence de quantification sur l'argument exprimé : chercher après ses clés ne se distingue pas de chercher ses clés par la quantité de clés requise pour satisfaire la description. L'AOO ne coïncide pas ici avec une différence de couverture référentielle relativement au procès signifié.

\subsection{Le schéma implicationnel}

Beavers (2006) formule une hypothèse destinée à rendre compte de l'ensemble des cas d'alternance de construction entre objets et obliques. Cette hypothèse ne se limite pas aux cas que nous avons identifiés pour le français, mais elle devrait a minima s'y appliquer. Tout en soulignant l'idiosyncrasie du phénomène observé, qui tient au fait que (i) d'une langue à l'autre, les $\mathrm{V}$ concernés par l'alternance varient, et que (ii) les V qui admettent l'alternance dans une langue donnée sont limités, y compris au sein d'une même classe sémantique, Beavers (2006: 280) suggère un principe général d'alignement morphosyntaxique, qui stipule que « direct argument variants imply more properties about the alternating participant than oblique variants». Autrement dit, la saillance morphosyntaxique des relations argumentales est décrite d'après un schéma d'implication unilatérale qui établit que, pour un même participant argumental, les effets décrits dans la construction directe impliquent ceux décrits dans la construction oblique, mais non réciproquement. Ainsi, selon Beavers, la construction directe comprend plus d'informations sémantiques que la construction oblique, et la dégradation syntaxique de l'objet correspond à la description d'un procès moins spécifié.

Ce schéma d'implication unilatérale est parfois vérifié en français. Les alternances conatives et exploratives, parce qu'elles impliquent dans la version directe une plus grande affectation du patient ou un parcours plus couvrant, valident l'hypothèse de Beavers. Tirer un lapin implique sémantiquement tirer sur un lapin, de même que fouiller l'appartement implique fouiller dans l'appartement, alors que dans les deux cas la réciproque est fausse. En outre, le schéma implicationnel rend compte de certains cas d'alternance non identifiés dans les travaux existants, tels que les doubles constructions des V travailler, penser, discuter, etc. :

(49) a. Comment avez-vous pensé le concept de l'hydrodiplomatie ? (web)

b. Quand on pense au concept de pauvreté, on pense immédiatement à la question des conditions de vie (web)

(50) a. J'ai travaillé l'idée d'un drapé couleur or avec une feuille de cire chauffée, et je suis arrivée à cette manchette en bronze mat avec un côté très texturé. (Le Monde, 10.02.2015)

b. L'artiste travaille sur l'idée que la photo aurait perdu sa fonction de mémoire, d'immortalité et de vérité. (Le Devoir, 10.06.2017)

La construction directe renvoie ici à un processus de création ou d'altération d'un objet cognitif, tandis que la tournure prépositionnelle s'en tient à l'évocation de cet objet. Aussi 
penser un concept, travailler une idée impliquent-ils unilatéralement penser à un concept, travailler sur une idée, ce qui est conforme à l'hypothèse d'un enrichissement de la description dans la structure directe.

Cette hypothèse, toutefois, ne suffit pas à rendre compte de l'ensemble des $\mathrm{AOO}$ observées en français. Même si l'on écarte les multiples alternances dans lesquelles il semble difficile de déceler une différence d'interprétation entre les deux constructions cas admis par Beavers $(2006: 65,142)$ et explicitement non pris en charge dans son modèle explicatif -, il existe de nombreux cas dans lesquels la variation sémantique observée ne correspond pas à une stricte inclusion du contenu informationnel de la construction oblique dans celui de la construction directe. Comme nous l'avons vu plus haut, la différence sémantique existant dans les alternances du type de grimper (sur) la colline tient essentiellement au profilage du site de localisation, et s'analyse difficilement comme un rapport unilatéral d'implication sémantique entre les deux constructions. Si grimper la colline implique grimper sur la colline, on peut également penser que la réciproque est vraie, et que grimper sur la colline inclut la référence à un parcours holistique. Par ailleurs, il arrive que l'implication sémantique entre les deux variantes de l'AOO semble être l'inverse de celle identifiée par Beavers. Tel est le cas dans les alternances de construction de toucher (à) et pousser $(\grave{a})$ :

\section{(51) a. Pierre a touché (à) l'écran.}

b. Le chat pousse (à) la porte.

Comme le montrent Bat-Zeev Shyldkrot \& Kemmer (1995: 212), toucher à , contrairement à toucher, présuppose l'intentionnalité de l'agent, ce qui permet différentes inférences sémantiques, concernant par exemple la manipulation de l'objet (Quelqu'un a touché à mon $b o l)$ ou l'itération de l'action décrite. La tournure prépositionnelle dans pousser à permet également d'exprimer l'aspect itératif et la pluriactionnalité, non nécessaires dans l'emploi direct. Il apparaît que la construction oblique dans (51) comporte plus d'information que la construction directe : le schéma implicationnel est bien unilatéral, mais inversé par rapport au cas prédit par l'hypothèse de Beavers.

\subsection{Vers une explication multifactorielle}

Les $\mathrm{AOO}$ en français paraissent relever de deux facteurs : la présence ou l'absence d'une différenciation sémantique et la présence ou l'absence de variation dia-(chronique, stratique, topique, phasique).

En cas de différenciation sémantique, plusieurs effets sont observés, suivant le type de procès en jeu : parcours, contact, élaboration / évocation, intentionnalité, etc. Comme nous l'avons vu, une partie des alternances observées n'est pas réductible aux alternances conative, locative et explorative. On peut faire l'hypothèse que la construction oblique présente dans certains cas, non pas une moindre implication sémantique, mais plutôt un écart par rapport à un schéma actanciel prototypique. L'oblicisation de l'objet correspondrait alors à une variation sémantique par rapport au schéma processif fondamental et aux relations entre participants décrits par défaut par le verbe - que la description concerne l'affectation du patient, la configuration spatiale, l'intentionnalité ou l'occurrentialité du procès. L'enrichissement de l'expression par ajout d'un élément prépositionnel coïnciderait de ce point de vue avec une complexification de l'information véhiculée quant à la situation décrite.

En cas d'absence de changement de sens clair (comme dans adjuger un tableau (pour) 100 millions, applaudir (à) le numéro des clowns), on a affaire à des effets de synonymie locale : 
(52) a L'œuvre, qui était estimée entre 600'000 et 800'000 euros, a été adjugée quelque $588^{\prime} 748$ francs, à Paris, samedi. (Tribune de Genève, 18.11.2017)

b. Ces dernières années, une dizaine de tableaux ont été adjugés pour 100 millions ou plus. (www.rts.ch, 16.11.2017)

Il ne semble en l'occurrence ni possible ni pertinent de déterminer l'orientation d'une quelconque implication sémantique entre la construction directe et la construction oblique.

Par ailleurs, certaines dimensions de la variation langagière compliquent sensiblement

la description. En effet, nombreuses sont les alternances qui s'expliquent (ou sont expliquées par des auteurs) par des usages différenciés, chez un même locuteur ou d'un locuteur à l'autre (selon son origine géographique, sa formation ou la strate chronologique dans laquelle il évolue).

Les facteurs de différenciation sémantique et de variation dia- sont orthogonaux, ce qui suppose quatre situations distinctes :

(i) Il existe des verbes pour lesquels on observe à la fois un sens différent et une variation, par exemple diachronique. C'est le cas de tirer (à) les corneilles et de fuir (de), où la construction prépositionnelle fait figure d'archaïsme (ainsi, fuir de est mentionné dans le Dictionnaire du Moyen Français, mais pas dans le TLFi).

(ii) Le cas le plus commun présente une variation dia-, mais sans corrélation sémantique. Ainsi, persuader $\grave{a} X$ et ressembler $X$ sont des archaïsmes, la forme standard étant désormais persuader $X$ et ressembler $\grave{a} X$. De même, Goosse-Grevisse (2016) signale le belgicisme renoncer un bail et écrit qu' " on dit au Québec contribuer mille dollars et, par un mouvement inverse, compenser pour qqch». Et on peut encore citer le germanisme attendre sur $X$, attesté entre autres en Suisse romande. La dimension diaphasique est elle aussi concernée, du moins si l'on en croit le Le Bon Usage qui voit des tours littéraires dans contredire au monstre et renoncer toute croyance, alors qu'attendre après ressortirait au registre familier.

(iii) Il existe également des alternances qui présentent une différenciation sémantique, sans toutefois que les dimensions de la variation entrent en jeu. C'est le cas de toucher (à), grimper (sur), tirer (sur), analysés supra.

(iv) Enfin, certaines alternances ne manifestent ni corrélat sémantique, ni dimension

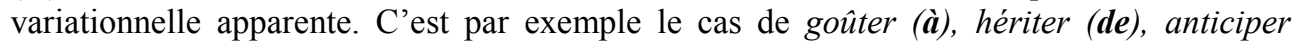
(sur).

En l'absence de variation sémantique, des alignements analogiques (ou présumés tels) peuvent expliquer l'émergence de certaines alternances, comme c'est le cas pour se rappeler de $x$, par analogie avec se souvenir de $x$, ou divorcer $X$ par analogie avec quitter $X$ (cf. Je l'ai divorcé, bien attesté sur le web). Le Bon Usage mentionne encore par exemple lorgner sur / vers (sur le modèle de loucher), attester de et préjuger de par analogie avec, respectivement, témoigner de et juger de. Voir également les exemples suivants :

(53) a. D'où cette question : André Kudelski a-t-il surestimé de ses forces ou a-t-il péché par excès d'optimisme? (La Liberté, 31.08.2002)

b. (...) les règles du monde auxquelles il doit suivre. (oral, recueilli 'à la volée' par un étudiant dans le cadre d'un travail universitaire, 2010)

On peut penser que surestimer de est construit par analogie avec présumer de et suivre $\grave{a}$ par analogie avec se conformer à ou obéir à. Pour toutes ces occurrences d'analogie présumée, la variation n'est pas motivée sémantiquement.

Notons enfin que les axes variationnels agissent souvent de conserve. Par exemple, dans l'alternance lui / l'empêcher, on pourrait voir une analogie avec la construction de l'un de ses antonymes (permettre $\grave{\boldsymbol{a}} X$ de faire), mais la construction dative (empêcher qqch $\grave{a}$ $\left.q q^{\prime} u n\right)$ "remonte au Moyen Age» selon Goosse-Grevisse ; et comme beaucoup d'archaïsmes (par exemple aider $\grave{a} X$ ), elle fait aujourd'hui figure de diatopisme. 


\section{Conclusion}

Bien que limitée à une micro-zone variationnelle de la syntaxe du français, notre recherche soulève des questions de portée plus générale. L'étude de l'AOO constitue une porte d'entrée possible sur la question des propriétés fondamentales assignables à la construction transitive, par rapport aux autres types de complémentation verbale. On peut également mentionner les deux aspects suivants :

(i) Si la concurrence entre les prépositions est aujourd'hui relativement bien documentée, on connaît moins bien la concurrence entre une préposition et son absence - ou entre un clitique accusatif et un clitique ablatif, par exemple (cf. l'/en abuser). Melis (2017: 29) signale une conséquence théorique possible :

L'hypothèse qu'il existe des prépositions incolores soulève toutefois une question théorique importante relative à l'existence de prépositions vides (...). L'existence de contrastes interprétatifs entre des paires telles que Ils habitent le quartier / dans le quartier. / Ils touchent le but / au but. (...) tout comme entre les diverses acceptions de croire suivi d'un groupe nominal nu ou d'un groupe introduit par $\grave{a}$ ou en, montre que la présence d'une préposition est significative et qu'elle est donc porteuse de sens, même s'il est parfois difficile d'en cerner la valeur précise.

(ii) Par ailleurs, plusieurs auteurs (Gougenheim 1970, Herslund 1997a, 1997b, Beavers 2006, Janic 2016) font état d'une parenté sémantique entre l'AOO et la construction antipassive (cf. agripper X/s'agripper à X, attaquer X/s'attaquer à X, saisir X/se saisir de $X$ ). Herslund (1997 : 79-80) rapproche explicitement l'AOO de l'antipassif :

Such alternations [i.e. celles qui relèvent de l'AOO] are lexically determined and not systematic grammatical choices, but they also exhibit the typical antipassive features: the marginalisation of the Patient and the loss of the object relation whereby the clause concentrates on the Agent. As mentioned above, the antipassive clause states that the Agent is involved in an activity which may be relative to a Patient, but this Patient is not integrated into the verbal situation with a central GR [grammatical relation]. It is the Agent's willful and intentional activity which is highlighted.

alors que Janic (2016), qui mentionne l'apparentement (ci-dessous), ne franchit pas le pas :

Une dernière perspective s'ouvrirait dès lors qu'on accepterait l'idée selon laquelle dans l'antipassif des langues accusatives, la présence du marquage verbal n'est pas pertinente. Cette démarche nous offrirait la possibilité d'inclure en français (mais aussi dans d'autres langues) les alternances de type GN1 V GN2 GN1 V à/de GN2 (...)

La réduction de nos alternances objet / oblique à l'alternance actif / antipassif, suppose que soient réglées une question formelle (l'antipassif implique-t-il une variation de la morphologie verbale ?) et une question de nature sémantique (la variation de sens observée dans les cas d'AOO est-elle comparable à celle identifiée dans les occurrences avérées de l'antipassif?) - questions qui ne peuvent pas être discutées dans le cadre de cet article.

\section{Références}

Adams C. (2001), The conative Alternation: An exploration of semelfactives and the elusive non-Theme Patient, M.A. thesis, University of Canterbury.

ATILF (laboratoire Analyse et traitement informatique de la langue française). Base textuelle Frantext. En ligne : http://www.frantext.fr/.

Bat-Zeev Shyldkrot H. \& Kemmer S. (1995), « La grammaticalisation des prépositions : concurrence et substitution », Revue romane 30-2, 205-225. 
Beavers J. (2006), Argument/Oblique Alternations and the Structure of Lexical Meaning, $\mathrm{Ph}$. D. thesis, Stanford.

Blanche-Benveniste C. (2010), Le français. Usages de la langue parlée, Leuven/Paris : Peeters.

Blinkenberg A. (1960), Le problème de la transitivité en français moderne, Copenhague: Munksgaard.

Bonami O. (1999), Les constructions du verbe : le cas des groupes prépositionnels argumentaux : analyse syntaxique, sémantique et lexicale, Thèse de doctorat, Paris 7.

Broccias C. (2001), "Allative and ablative at-constructions », CLS 37, 67- 82.

Creissels D. (2006), Syntaxe générale. Une introduction typologique, Paris : Hermès.

Danlos L. (1986), «Une illustration d'étude formelle des noms : charg(-e, -er, -ement) », Langue Française 69, 28-48.

Delhem R. (2015), "Schémas argumentaux et homonymie : l'exemple de la construction conative en anglais ». ELIS - Echanges de linguistique en Sorbonne 3-2. En ligne: http://elis.hypotheses.org/313.

Eynde K. van den \& Mertens P. (2003), «La valence: l'approche pronominale et son application au lexique verbal », Journal of French Language Studies 13, 63-104.

Fillmore C. (1968), "The Case for Case », Universals in Linguistic Theory. Bach E. \& Harms R. T. (ed.), New York: Holt, Rinehart, and Winston, 1-88.

Franckel J.-J. \& Paillard D. (2007), Grammaire des prépositions, Tome 1, Paris : Ophrys. Goosse-Grevisse ( $\left.{ }^{16} 2016\right)$, Le Bon Usage, De Boeck-Duculot.

Gougenheim G. (1970), Etudes de grammaire et de vocabulaire français, Paris : Picard.

Goyens M. (1998), «L'alternance entre construction accusative et dative dans l'histoire des verbes français », Leuvense bijdragen 87 (3-4), 465-489.

Goyens M. (2000), «L'alternance dans les constructions prépositionnelles des verbes français : une étude historique de l'emploi de la préposition à », Les nouvelles ambitions de la linguistique diachronique, Tübingen : Niemeyer, 203-210.

Goyens M. (2001), "L'origine des verbes français à construction dative », La valence, perspectives romanes et diachroniques, Schosler L. (éd.), Stuttgart : Steiner.

Guillet A. \& Leclère C. (1992), La structure des phrases simples en français. Les constructions transitives locatives, Genève : Droz.

Herslund M. (1997a), "Syntaktiske alternationer og funktionelle kategorier », Falster Jakobsen L. \& Skytte G. (éds). Ny Forskning i Grammatik 4, 49-70.

Herslund M. (1997b), «Passive and Antipassive in a Functional Description of French Reflexive Verbs », Hermes. Journal of Linguistics 19, 75-92.

Janic K. (2016), L'antipassif dans les langues accusatives, Bruxelles : Peter Lang.

Lamiroy B. \& Charolles M. (2011), «Des formes aux sens: les clitiques dans la construction causative en faire ", Du système linguistique aux actions langagières, Corminboeuf G. \& Béguelin M.-J. (éds), 41-59.

Leek F. van der (1996), "The English Conative Construction: A Compositional Account », Papers from the Chicago Linguistic Society 32, 363-378.

Levin B. (1993), English Verb Classes and Alternations: A Preliminary Investigation, Chicago: University of Chicago Press.

Melis L. (2017), «La préposition », Encyclopédie Grammaticale du Français. En ligne : http://encyclogram.fr

Perek F. \& Lemmens M. (2010), «Getting at the meaning of the English at-construction: the case of a constructional split», CogniTextes 5. En ligne : http://cognitextes.revues.org/331.

Picoche J. (1986), Structures sémantiques du lexique français, Paris : Nathan.

Prandi M. (1987), Sémantique du contresens, Paris : Minuit.

Riegel M., Pellat J-C. \& Rioul R. (1994). Grammaire méthodique du français, Paris, PUF. 
Sablayrolles J.-F. (2011), «De la 'néologie syntaxique' à la néologie combinatoire », Langages 183, 39-50.

Sosa Acevedo E. (2011), "Setting up the boundaries for the conative construction in Spanish », RESLA 24, 227-255.

Talmy L. (2000), Toward a Cognitive Semantics, Cambridge MA : MIT Press.

Willems D. (1981), Syntaxe, lexique et sémantique. Les constructions verbales, Gand : Publications de l'Université de Gand.

${ }^{1}$ Nous remercions Frédéric Gachet pour sa contribution à l'élaboration des hypothèses présentées ici, Clément Plancq pour son aide technique dans l'extraction des données pertinentes de Dicovalence et les deux relecteurs anonymes pour leurs commentaires sur la première version de l'article.

${ }^{2}$ La voix est comprise ici comme un encodage morphologique de la valence verbale (cf. Creissels 2006). La condition de préservation de la voix dans l'AOO est destinée à écarter a priori les cas d'oblicisation de l'objet liés à un changement de morphologie verbale, tels que ceux induits par l'emploi d'un antipassif en se $V$ en français (ex. agripper le volant / s'agripper au volant, cf. Janic 2016).

${ }^{3}$ Les $\mathrm{V}$ descendre et monter ne sont pas pour autant exclus des $\mathrm{V}$ qui présentent une AOO, étant donné qu'il existe des emplois (non standard) de ces $\mathrm{V}$ avec l'auxiliaire avoir, comme dans $O n$ a descendu de la montagne à vélo et maintenant on doit remonter à pied (web), ainsi qu'avec l'auxiliaire être comme dans Ils étaient sortis de chez eux, ils étaient descendus le perron (Ramuz, Passage du poète). Cette variation peut rendre indistincts les cas d'alternance et de non alternance lorsque les $\mathrm{V}$ concernés sont employés aux temps non composés.

${ }^{4}$ L'implication inverse n'est pas vérifiée : ce n'est pas parce qu'un verbe se construit avec plusieurs prépositions que le SP n'a pas un statut d'argument (ex. habiter \{ à / dans / sur / près de / au nord de / etc.\} $S N)$.

${ }^{5}$ Au sens de : charger (sur / contre) les manifestants.

${ }^{6}$ A l'inverse, la possibilité d'insérer le SN argumental un coup de feu dans l'expression tirer sur un renard n'exclut pas tirer (sur) un renard des candidats à l'AOO, étant donné que l'objet n'a pas le même rôle sémantique dans tirer un coup de feu et tirer un renard.

${ }^{7}$ Il arrive même que des SP qui, à première vue, ne sont ni des arguments syntaxiques ni des arguments sémantiques alternent avec des SN objets dans des tournures transitives. Tel est le cas lorsque le V aboyer est suivi d'un SN au lieu d'un SP introduit par sur ou après: Ma voisine m'a prévenu qu'il n'avait pas arrêté d'aboyer o les passants dans la cage d'escalier (web). L'existence d'alternances de ce type, fussent-elles rares et marquées, interroge in fine sur les ressorts de la transitivité et les conditions d'accès au statut argumental pour un constituant donné.

8 Beavers (2006) distingue plus précisément deux types d'alternance conative, selon que la spécification porte sur (i) l'affectation vs la non-affectation de l'objet (ex. John slashed (at) the canvas 'John a lacéré (/ a tenté de lacérer) la toile'), ou (ii) sur l'affectation totale vs partielle de l'objet (John ate (at) the sandwich 'John a mangé (en partie) le sandwich'). Dans les deux cas, la construction directe se distingue de la construction prépositionnelle par le degré d'aboutissement de l'action décrite.

${ }^{9}$ Voir Broccias (2001) pour une critique circonstanciée de l'approche de Levin.

${ }^{10}$ On infère généralement de la construction tirer $+S N$ que le patient de l'action est abattu par le coup de feu. Cette interprétation ne paraît toutefois pas nécessaire, comme en atteste l'exemple suivant: Un officier du 10e hussards avait, au début d'une battue dans les bois d'une chasse de commune, tiré un renard sans résultat appréciable. Le renard s'était dérobé, comme d'habitude, dans le fourré d'un bois feuillu montant au long d'une pente. (J.-C. Hermanns, Google Livres).

${ }^{11}$ Beavers pointe de la même manière la différence existant entre la postposition -ni en japonais et la particule up en anglais. La première indique le but, ce qui implique que dans la construction oblique du japonais Yama-ni nobotta 'Je suis monté sur la montagne', le sommet de la montagne soit atteint, contrairement à ce qu'on observe pour l'anglais. 